\title{
Recent Experimental Results from Spectroscopy of Superheavy Quasiatoms
}

\author{
P. Armbruster, G. Kraft and P. Mokler \\ GSI, Darmstadt \\ B. Fricke \\ Gesamthochschule Kassel und GSI, Darmstadt
}

H. J. Steîn

Institut für Kernphysik, KFA Jülich

Received July 2, 1974

\begin{abstract}
Recent experimental results from spectroscopy of superheavy quasiatoms. $P$. Armbruster, G. Kraft, P. Mokler (GSI, Darmstadt, West Germany), B. Fricke (Gesamthochschule Kassel und GSI, Darmstadt, West Germany) and H. J. Stein (Institut tür Kernphysik, KFA Jülich, West Germany). Physica Scripta (Sweden) 10 A, 175-182, 1974.

The concept of a "Superheavy Quasiatom" is discussed. Radiative transition times are compared with the lifetime of the intermediate system, cross sections are calculated within a two-collision model and induced transitions and their anisotropic emission are discussed. Recent experimental and theoretical results are presented from collision systems obtained with $I$-beams bombarding various heavy targets, giving combined $Z$-values between 120 and 145. Results include the energy dependence of the peak structure interpreted as $M$ X-rays from superheavy quasiatoms and the anisotropy of $\mathrm{X}$-ray emission referred to the beam direction. The data are discussed within the models available. These cannot explain the strong emission of anisotropic radiation in the $\mathrm{X}$-ray energy range of quasiatomic $M \mathrm{X}$-rays at small bombarding energies.
\end{abstract}

\section{Introduction}

Non-characteristic X-rays emitted in collisions between heavy ions and heavy targets leading to a combined nuclear charge larger than the charges of known elements have been investigated in the last two years $[1,2,3,4]$. The experiments have been performed at the Tandem Accelerator of the University of Cologne. The experimental set-up used is shown in Fig. 1. It is described in detail in refs. [1] and [3]. A spectrum obtained in Au-bombardments by $11 \mathrm{MeV}$ J-ions is shown in Fig. 2. A broad structure is found in the X-ray spectrum at energies between the characteristic $L$ X-rays of iodine and gold. This structure has been attributed to $X$-rays from a transient combined system with an effective charge $Z=132$ equal to the sum of the charges of $J+A u$ [1]. In the following the general ideas and recent experimental results are discussed. These support the interpretation formerly given.

\section{General remarks concerning the concept of a "Superheavy Quasiatom"}

1.1. Let the sum $Z$ of the two nuclear charges $Z_{1}$ and $Z_{2}$ of the collision partners be larger than the natural atomic numbers. For these cases the combined charge equals the charge of the superheavy element.

$Z=Z_{1}+Z_{2} \geqslant 105$
1.2. A minimum distance of closest approach $b$ is necessary to make the Coulomb fields of the collision partners appear to the electrons at a distance $a_{n}$ as the Coulomb field of a combined system.

$b_{\min } \leqslant a_{\mathrm{n}}$

For $b$ smaller than $a_{\mathrm{n}}$ the energy values of the transient system are assumed not to change any more. They are set equal to the values of the combined system. Eq. (2a) is equivalent to a minimum value of the collision velocity $v$,

$\frac{v}{v_{0}} \geq 2 \times 10^{-2} \frac{Z}{n}$

with $v_{0}=\alpha c$, and $n$ the principal quantum number. This minimum velocity is necessary to build the transient superheavy system.

1.3. The eigen-times of the combined system must be smaller than the life-time of the system. Then the transient system may be called a "quasiatom".

$\frac{n h}{E_{n}} \leqslant 2 \frac{a_{n}}{v}$

There is a maximum velocity up to which the quasiatom picture is applicable. Above this velocity there is no time to develop well defined electron orbits, an indispensable characteristic of an atomic system.

$\frac{v}{v_{0}} \leqslant 0.16 \frac{Z}{n}$

The minimum velocity of eq. ( $2 b$ ) and the maximum velocity of eq. ( 3 b) have a ratio

$\frac{v_{\min }}{v_{\max }} \sim 0.13$

which is independent of the atomic number or the shell. For all electron shells there is a velocity range, in which the transient system may be considered as a "Quasiatom".

1.4. "Life-time of the quasiatom" and "collision time" are used as synonyms. The life-time falls to zero both for very small as well as for large values of the velocity. For small values of $v$ there is no overlap of the two colliding systems, for large values of $v$ the 


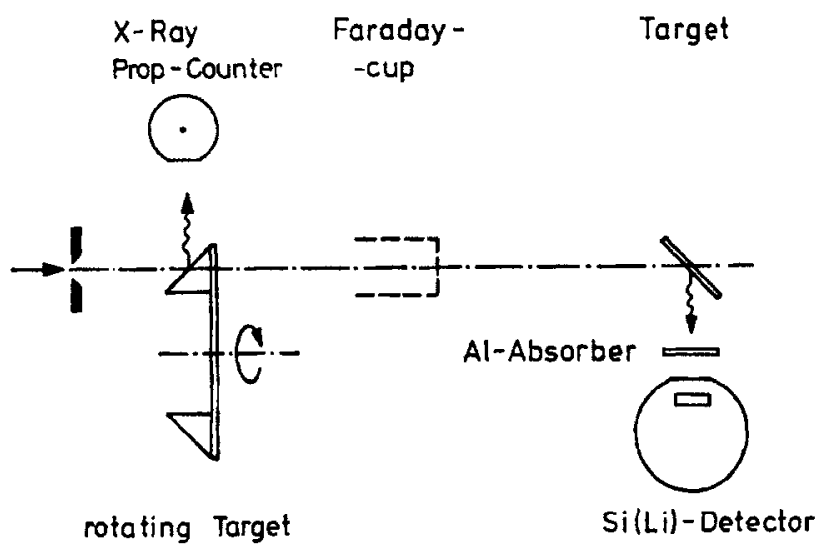

Fig. 1. Experimental set-up to determine absolute X-ray production cross sections in heavy ion collisions (ref. [3]).

collision time falls to zero as $1 / v$. An optimum distance of closest approach exists giving the longest possible life-times of the quasiatom.

$b_{\text {opt }}=0.61 a_{n}$

$T_{\text {coll }} \leqslant T_{\max }=10^{-16} \frac{n^{2}}{Z^{2}}[\mathrm{~s}]$

with

$v_{\text {opt }} \sim v_{\min }$

The largest life-times of quasiatoms are obtained near the lowest possible velocity (see 1.2).

1.5. Information about states of the combined quasiatomic system may be obtained, if electromagnetic transitions from the system can be observed during its life-time. Electromagnetic transitions are due to the interaction

$H_{\mathrm{rad}}=j A$

with $A$ the vector potential of the field, and $j$ the electromagnetic current.

1.5.1. The vector potential $A$ of the photonfield within the quasiatom is assumed to be represented by a plane wave:

$A=\varepsilon \mathrm{e}^{\mathrm{i}(\omega t-k r)} \sim\left[1-i(k r)-\frac{1}{t}(k r)^{2} \ldots\right] \mathrm{e}^{-t_{\omega} t}$

with $\varepsilon$ the polarisation vector and $\boldsymbol{k}$ the wave number of the plane wave.

$\omega t=\omega T_{\text {ool1 }}<2 \pi \cdot n$

$|k r|=\frac{2 \pi}{\lambda} a_{\mathrm{n}}=\frac{\alpha Z}{2}<0.7$

$|\boldsymbol{k r}|$ is not small for superheavy quasiatoms. The transition probabilities of higher multipolarities will be enhanced.

1.5.2. Spontaneous radiative E1-transitions are caused by the spontaneous electronic current [5].

$\dot{j}_{\text {spon }}=\dot{e r}$

The life-time for E1-transitions follows

$\tau_{n n E_{1}}=\frac{\tau_{0}}{\omega_{m n}^{8}\left|D_{m n}\right|^{2}}=4 \times 10^{-10} \frac{n^{2}}{Z^{4} k_{n}^{2}}[\mathrm{~s}]$

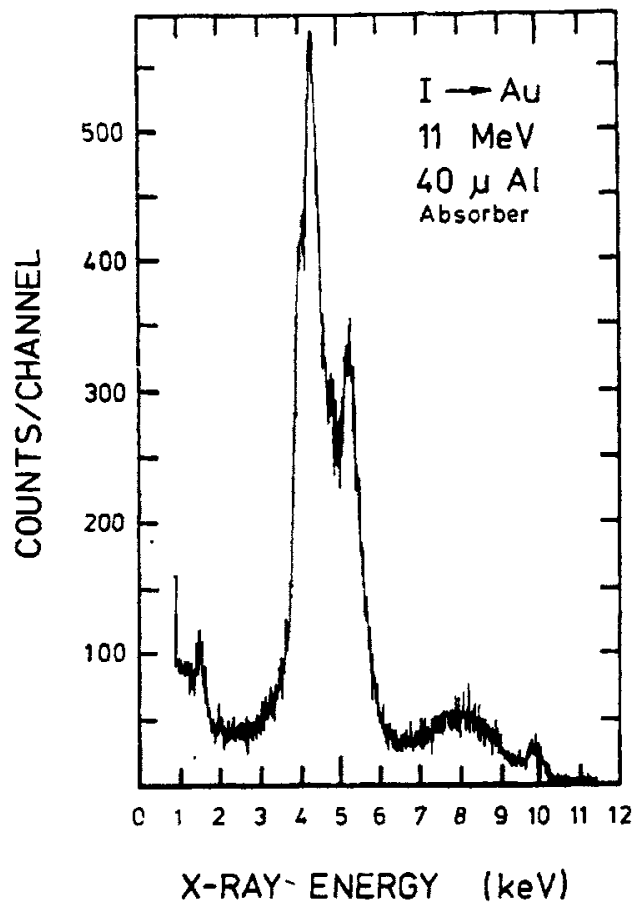

Fig. 2. X-ray spectrum obtained from $\mathrm{I} \rightarrow \mathrm{Au}$ collisions at $11 \mathrm{MeV}$ bombarding energy (ref. [1]).

with

$k_{n}=\frac{2 n+1}{(n+1)^{2}}$

The ratio collision time to radiative transition time equals:

$\frac{T_{\text {coll }}}{\tau_{m n \mathrm{E} 1}^{\mathrm{rad}}} \leqslant 0.8 \times 10^{-6} Z^{2}$

In very heavy systems the ratio becomes 0.03 , whereas in nuclear systems it is $10^{-7}$. X-rays from quasiatoms should be enhanced in heavy systems. It is still an open question how much higher multipolarities contribute.

1.5.3. B. Müller et al. [5] have shown how the collision induced transitions $[1,3]$ may be taken into account. They are due to the current produced by the rotation of the intermediate system around the internuclear axis.

$H_{\text {1nd }}=j_{\text {rot }} \cdot A$

with $J_{\text {rot }}-e\left[\omega_{\text {rot }} \cdot r\right]$

The rotational velocity $\left[\omega_{\text {rot }} \cdot r\right]$ is determined by the position vector $r$ of the electron and the angular velocity $\omega_{\text {rot }}=l / \mu R(t)^{2}$ of the molecular system, where $l$ is the angular momentum of the two colliding nuclei and $\mu R^{2}$ their moment of inertia at a distance $R(t)$. The transition probability for rotational induced transitions is:

$\tau_{m n \mathrm{EI}}^{\mathrm{ind}}-\frac{\tau_{0}}{\omega_{m n} \omega_{\text {rot }}^{2}\left|D_{m n}\right|^{2}}-\frac{\omega_{m n}^{2}}{\omega_{\text {rot }}^{2}} \tau_{m n \mathrm{EI}}^{\mathrm{rad}}$

For small impact parameters, as well as for large impact parameters $\omega_{\text {sot }}$ falls to zero. $\omega_{\text {rot }}$ has a maximum value for a certain impact parameter, to which corresponds a given minimum distance of approach. Here the maximum value of $\omega_{\text {rot }}$ is obtained.

$\left|\omega_{\mathrm{rot}}\right|<0.35 \frac{v}{b}=3.3 \times 10^{19} \frac{1}{Z}\left(\frac{v}{v_{0}}\right)^{3}\left[\mathrm{~s}^{-1}\right]$ 
At large values of $v$ the induced transitions become dominant, whereas at small velocities the spontaneous transitions prevail.

$\left|\omega_{\text {rot }}\right|-\omega_{m n}$ for $\frac{v}{v_{0}} \sim 9 \times 10^{-2} \frac{Z}{n}$

As long as the minimum distance of approach corresponding to $\omega_{\text {rotmax }}$ is smaller than $a_{n}$, the shell radius of the quasiatom, the energy of the induced transitions will mainly be found at the energy values of the quasiatom. Only for bombarding energies near the lower energy limit, eq. ( $2 \mathrm{~b}$ ), will the induced transitions appear at transition energies corresponding to the molecule.

1.6. Radiative transitions in quasiatoms are possible if a vacancy decays in an inner electronic shell. The question to be investigated now is, how do we produce vacancies in inner shells of quasiatoms? Saris et al. [6] proposed a two-collision mechanism to produce $X$-rays from quasiatoms. In a first collision a vacancy is produced in an inner shell of the projectile. This vacancy is transferred during a second collision happening within its lifetime to the combined system where it decays or is transferred after the collision into either of the collision partners.

Vacancy production in heavy ion collisions is possible in the adiabatic region mainly by electron promotion and level crossing in the molecular system [7,8]. Experiments show that this molecular excitation mechanism strongly dominates over Coulomb excitation [9-11]. Non-radiative interactions between different states at level crossings are possible. The kinetic energy of the two colliding atoms leads to a radial coupling at the crossing points with $\Delta m=0$. The transition probability is described by the Landau-Zener formula. The rotation $\omega_{\text {rot }}$ leads to a Coriolis interaction- $\omega_{\text {rot }} J$ with $J$ the orbital angular momentum of the electron. This rotational coupling occurs at all crossing points, as $\Delta m$ can be changed by transferring angular momentum from the orbital momentum of the two nuclei to intrinsic orbital momentum of the electrons.

To produce a large number of vacancies in the first collision, electron promotion to higher levels is used, e.g. to produce vacancies in the $L$-shell of a projectile which is lighter than the target atom, promotion to the $4 f$-level in the combined system is taken advantage of. In the second collision the vacancies in the $L$-shell of the projectile are transferred from its $2 p$-level via the $3 d$-level of the combined system into the $2 p$-level of the target atom (see Fig. 4). On the way from the $3 d$-level to the $2 p$-level a crossing has to be passed. Here a radial coupling will occur which allows the vacancy to jump over the crossing point.

The probability of finding X-rays from the quasiatom depends on (1) the cross section to produce primary vacancies in the projectile $\sigma_{1}$; on (2) the survival probability of the vacancy until the second collision

$\underline{\tau_{m n 1}^{\mathrm{rad}}}$

$\tau_{12}$

with $\tau_{m n 1}^{\mathrm{rad}}$ being the spontaneous radiative life-time of the vacancy in the projectile, and $\tau_{12}$ the average time between the two collisions; and on (3) the ratio between the collision time, that is the life-time of the quasiatom, and the radiative life-time of a vacancy in the quasiatom

$\frac{\sigma_{m n Z}}{\sigma_{1}}=\frac{\tau_{m n 1}^{\mathrm{rad}}}{\tau_{12}} \frac{T_{\mathrm{col1}}}{\tau_{m n}^{\mathrm{rad}}}$

Putting $\tau_{12}=1 /\left(v N \pi a_{n}^{2}\right)$ with $N$ the number of atoms per $\mathrm{cm}^{3}$, and taking $T_{\text {coll }}$ from eq. (4), we get the cross section for spontaneous transitions in the quasiatom:

$\frac{\sigma_{m n z}^{\mathrm{spon}}}{\sigma_{1}}-\frac{\tau_{m n 1}^{\mathrm{rad}}}{\tau_{m n}^{\mathrm{rad}}} N 2 \pi a_{n}^{3} \approx 0.5 \frac{n^{6}}{Z^{3}}$

The induced transitions depend on the value of $\omega_{\text {rot }}^{2} \tau_{\text {rot }}$, which has its maximum at $R_{\mathrm{min}}=2 b$.

One obtains with $T_{\text {cond }}^{\text {ind }}=\frac{2 R_{\min }}{v}=\frac{4 b}{v}$, and eq. $(8 \mathrm{~b})$;

$\frac{\sigma_{m n Z}^{\mathrm{ind}}}{\sigma_{1}}=\frac{\sigma_{m n Z}^{\mathrm{spon}}}{\sigma_{1}} \frac{\omega_{\mathrm{rot}}^{2} T_{\mathrm{coll}}^{\mathrm{ind}}}{\omega_{m n}^{2} T_{\mathrm{coll}}^{\mathrm{spon}}} \approx 7.7 \times 10^{3}\left(\frac{v}{v_{0}}\right)^{4} \frac{n^{8}}{Z^{7}}$

The production of X-rays in the quasiatom is density dependent. In monoatomic gas targets it should be strongly depressed. As $\sigma_{2}$ as well as $\sigma_{m n z}$ depends strongly on the atomic shell, we decided early [1] to look for quasiatomic X-rays in the $M$-shell of superheavy systems.

$\sigma_{M}: \sigma_{L}: \sigma_{K}=10^{6}: 10^{2}: 1$

$\sigma_{m n z} / \sigma_{1}$ does not depend on the velocity for spontaneous radiative transitions, whereas it increases with increasing velocity for induced radiative transitions. The ratio of cross sections for spontaneous and induced transitions is given by:

$\frac{\sigma^{\text {dnd }}}{\sigma^{\text {spon }}}=1.7 \times 10^{3}\left(\frac{v n}{v_{0} Z}\right)^{4} \geqslant 3 \times 10^{-4}$

For $v / v_{0}=0.16(Z / n)$, at the end of the adiabatic region, the two cross sections become equal. For higher velocities the induced radiation should dominate. But as already for $v / v_{0}=2\left(v_{\mathrm{min}} / v_{0}\right)>3 \times$ $10^{-2}(Z / n)$ the induced transitions will appear at the transition energies of the quasiatom, induced transitions will contribute mainly to the radiation found at quasiatom energies.

1.7. Angular distribution. The angular distribution of spontaneously emitted $\mathrm{X}$-rays is determined by

$\left(\frac{\mathrm{d} \sigma}{\mathrm{d} \Omega}\right)_{\mathrm{spon}}>\left|\varepsilon D_{m n}\right|^{2}$

As the polarisation $\varepsilon$ of the photons as well as the electric dipole moment are assumed not to show any preferential directions, the spontaneous radiation from the separated system and the quasiatomic system is expected to be emitted isotropically.

The induced transitions are depending on the alignment of the angular velocity vector $\omega_{\text {rot }}$. The alignment leads to an anisotropy of induced El-transitions corresponding to a radiating dipole in beam direction. As B. Müller et al. [5] have shown, the following expression holds true:

$\left(\frac{\mathrm{d} \sigma}{\mathrm{d} \Omega}\right)_{\mathrm{ind}} \sim\left|\varepsilon\left[\frac{\omega_{\mathrm{rot}}}{\left|\omega_{\mathrm{rot}}\right|} \times D_{\mathrm{mn}}\right]\right|^{\mathrm{a}}$

giving

$\left(\frac{\mathrm{d} \sigma}{\mathrm{d} \Omega}\right)_{\mathrm{Ind}} \sim \sigma_{\mathrm{ind}}\left(1+\frac{1}{2} \sin ^{2} \theta\right)$

with $\theta$ the angle between the photon and the beam diredtion. The anisotropy allows to differ between induced and spontaneous radiative transitions, if $\varepsilon$ and $D_{m n}$ do not have preferential direc- 


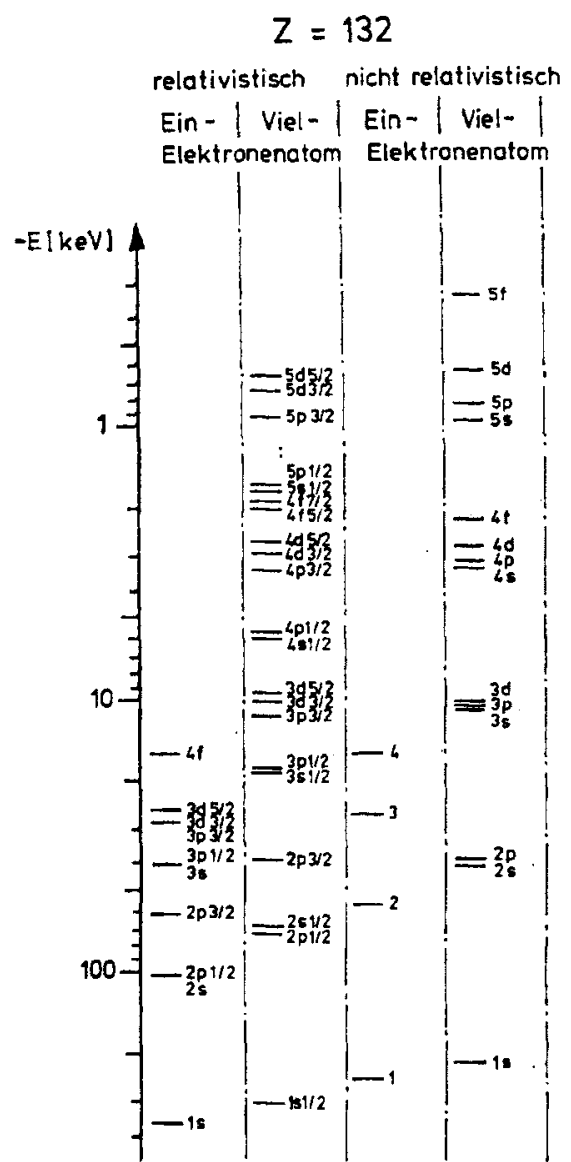

Fig. 3. Energies of the atomic states of element $Z-132$, calculated within different approximations (ref. [12]).

tions. From the anisotropy $k$, defined as $\sigma(\theta)=\sigma\left(1+k \sin ^{2} \theta\right)$, we get:

$\frac{\sigma_{\text {ind }}}{\sigma_{\text {apon }}}-\frac{1}{\frac{1}{2 k}-1}$

The anisotropy increases with increasing contribution of induced transitions, that is, with increasing collision velocity. The softer part of the quasi-molecular radiation should be less anisotropic, as it contains a higher amount of spontaneous transitions. As the induced transitions mainly appear at distances in the quasiatomic region, the anisotropy should be largest at the quasiatom limit. The induced radiation will be liniarly polarized in direction of the beam, a direct consequence of the anisotropic emission.

\section{The J-Au system as an example of a superheavy} quasiatom with $Z=132$

B. Fricke [12] has calculated the energy values of the electronic states of $Z=132$ (Fig. 3). Many-electron effects govern the se-

$$
\text { Au - J }
$$

20 Elektroden relativistisches Vielelektronen Korrelationsdiagramm

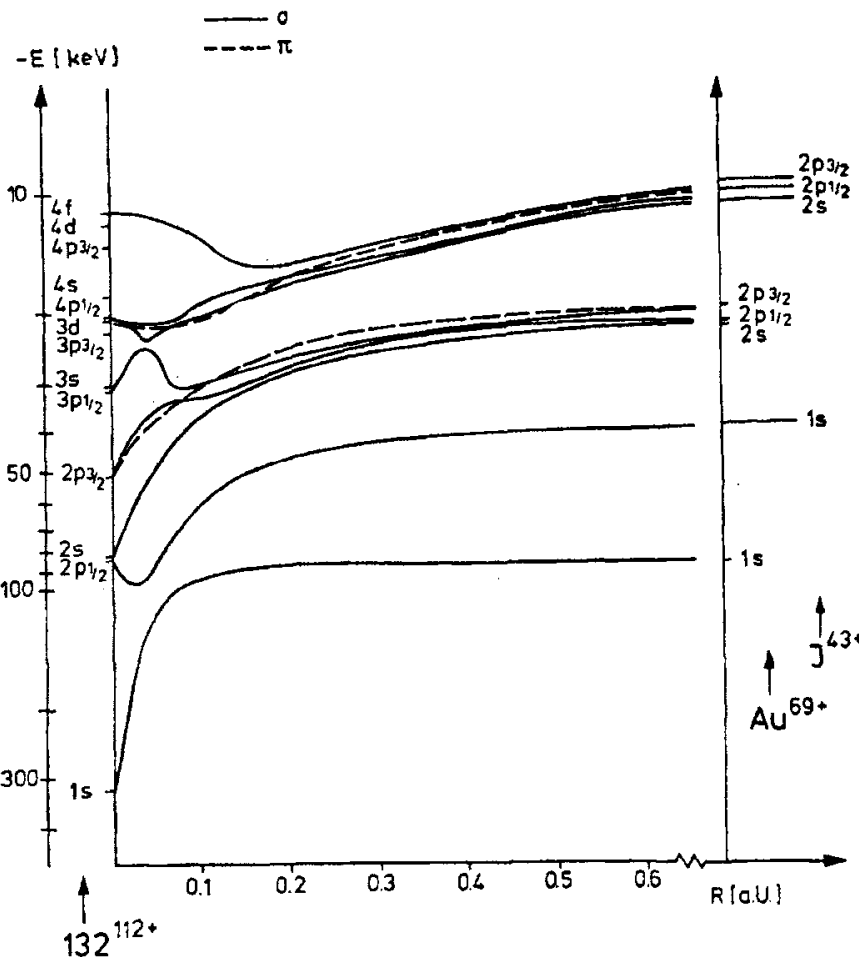

Fig. 4. Correlation diagram for the $\mathrm{I} \rightarrow$ Au system taking into account relativistically 20 electrons.

quence of states in the outer shells, whereas relativistic effects prevail for the $K$-shell. A relativistic correlation diagram taking into account 20 electrons is presented in Fig. 4. A Landau-Zener crossing at a distance $2 \times 10^{-10} \mathrm{~cm}$ is clearly seen between the $3 p \frac{1}{1} \mathrm{z}^{-}$and $3 d \frac{9}{2}$-states. The correlation diagram describes the static non-rotating molecular system. A rotating system would show interactions at the crossings where rotational coupling becomes possible. In Table I we present the most important quantities which have been discussed in section 1 for X-rays from the different main shells of $Z=132$.

It is evident that the low cross section for production of quasiatomic $K \mathrm{X}$-rays, $\sigma<1 \mu \mathrm{b}$, and not the energy of available heavy ion beams $E>0.2 \mathrm{MeV} / \mathrm{AMU}$ is the main experimental difficulty. An experiment to observe quasiatomic X-rays from the $L$-shell of element $Z=132$ could be done in the energy range of (0.1-2) MeV/AMU. The cross section of $10 \mathrm{mb}$ is comparablu to experiments done already with quasiatomic $K$ X-rays of lighter systems $(Z<70)[13-15]$.

The experiments investigating the $M$-shell of superheavy quasiatoms have large cross sections at energies easily available at

Table 1. Relevant data for the $J+A u(Z=132)$-system

\begin{tabular}{|c|c|c|c|c|c|c|c|c|c|}
\hline & \multirow{2}{*}{$\frac{E_{\text {min }}}{\mathrm{MeV}}$} & \multirow{2}{*}{$\frac{E_{\max }}{\mathrm{MeV}}$} & \multirow{2}{*}{$\frac{T_{\text {coll max }}}{10^{-1 g_{s}}}$} & \multirow{2}{*}{$\frac{\tau_{\mathrm{rad}}^{\mathrm{spon}}}{10^{-17_{s}}}$} & \multirow{2}{*}{$\frac{\sigma_{z}^{\text {apon }}}{\sigma_{1}}($ eq. 11 a) } & \multicolumn{2}{|c|}{$\left(\frac{\sigma_{z}}{b}\right)_{E-4 E_{\min }}$} & \multicolumn{2}{|c|}{$\left(\frac{K}{\%}\right)_{E-4 E_{\min }}$} \\
\hline & & & & & & calc. & measured & calc. & measured \\
\hline $\begin{array}{l}K \text {-shell } \\
L \text {-shell } \\
M \text {-shell }\end{array}$ & $\begin{array}{l}27 \\
12 \\
3\end{array}$ & $\begin{array}{r}1440 \\
640 \\
160\end{array}$ & $\begin{array}{c}0.2 \\
1.2 \\
15\end{array}$ & $\begin{array}{l}0.28 \\
2.6 \\
12\end{array}$ & $\begin{array}{l}22 \times 10^{-7} \\
14 \times 10^{-6} \\
15 \times 10^{-4}\end{array}$ & $\begin{array}{c}10^{-5} \\
10^{-2} \\
1\end{array}$ & $\overrightarrow{50}$ & $\begin{array}{l}0.73 \\
0.32 \\
0.24\end{array}$ & $\begin{array}{l}- \\
50\end{array}$ \\
\hline
\end{tabular}




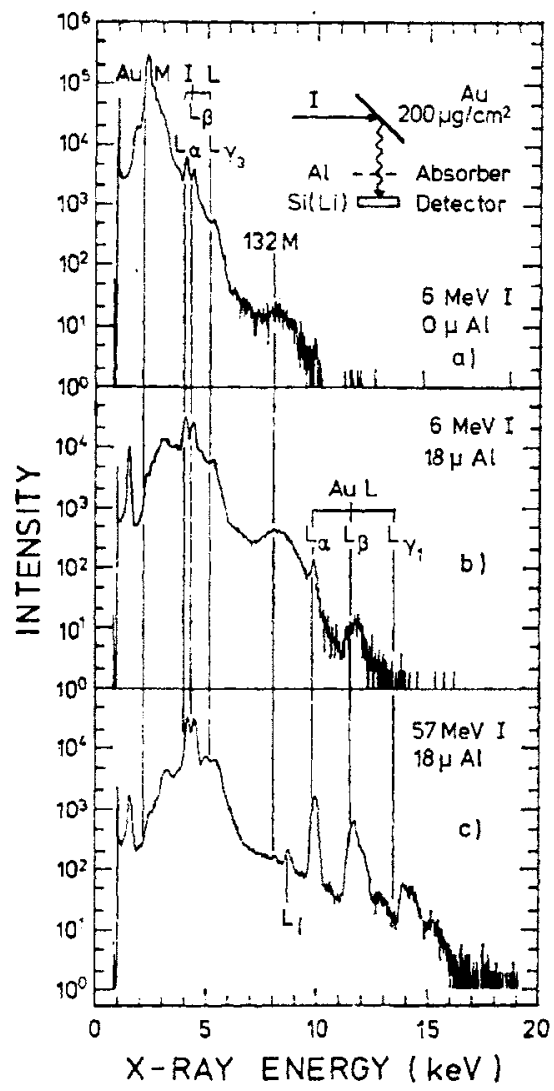

Fig. 5. X-ray spectra from $I \rightarrow$ Au collisions.

Tandem accelerators. These high cross sections allow detailed experimental studies in reasonable times. The phenomenon can thus be investigated thoroughly. Total as well as differential cross sections, anisotropies, energy dependences, in a wide range of atomic numbers from 120-150 can be covered.

\section{Experimental results}

During the last two years our first experimental results, ref. [1], have been checked carefully. They have been confirmed by an independent group [4]. The most striking result found was the peak structure at about $8 \mathrm{keV}$ and its high production cross section (see Fig. 2). In the measurements presented a high resolution $\mathrm{Si}(\mathrm{Li})$-detector and thin targets are used. Care is taken to prevent pile-up effects in the energy range of the structure to be investigated. The absorbers used to absorb the low-energy part of the spectrum have been optimized. Fig. 5 shows a spectrum taken with no absorbers with negligible pile-up at $6 \mathrm{MeV}$. The peak structure does not disappear. Experimentally the structure is real. Different possibilities to produce high energy X-rays, which have nothing in common with the mechanism discussed, had to be considered and calculated. Bremsstrahlung from high energy electrons produced in the collision, as well as nuclear bremsstrahlung contribute to a high-energy tail of X-ray lines. Electron capture from the continuum into the projectile leads to radiation at energies higher than the characteristic X-rays. All effects mentioned are definitely not responsible for the structure at $8 \mathrm{keV}$. No other plausible interpretation can be offered as quasiatomic $\mathrm{X}$-rays from a superheavy transient system.

Figs. 6 and 7 give spectra at different bombarding energies for gold and lead targets. The peak structure is seen best at the lowest energy. It broadens and disappears at about $40 \mathrm{MeV}$. Similar spectra have been obtained for Th- and U-targets. In Fig. 8 we give the energy of the peak position for various targets. Fig. 9 gives the width of the peak and the number of counts in the peak relative to the number of counts in the projectile $L$-lines depending on the bombarding energy. Fig. 10 gives the absolute cross sections and their energy dependence for the $L$-lines of the collision partners and the quasiatomic $M$ X-rays. Fig. 11 finally shows the result of a first anisotropy measurement.

\section{Discussion of results}

The peak position of the quasiatomic structure is compared with the energy values of the $(4 f-3 d)$-transitions obtained by relativistic, many electron calculations [12], (Fig. 8). This transition is expected to be observed, as the primary vacancies in the projectile are $2 p 3 / 2$ vacancies, which are transferred to the $3 d$-level of the combined"system. Only via the $3 d$-level the $2 p 3 / 2$ level of the target can be reached. The agreement between measured and

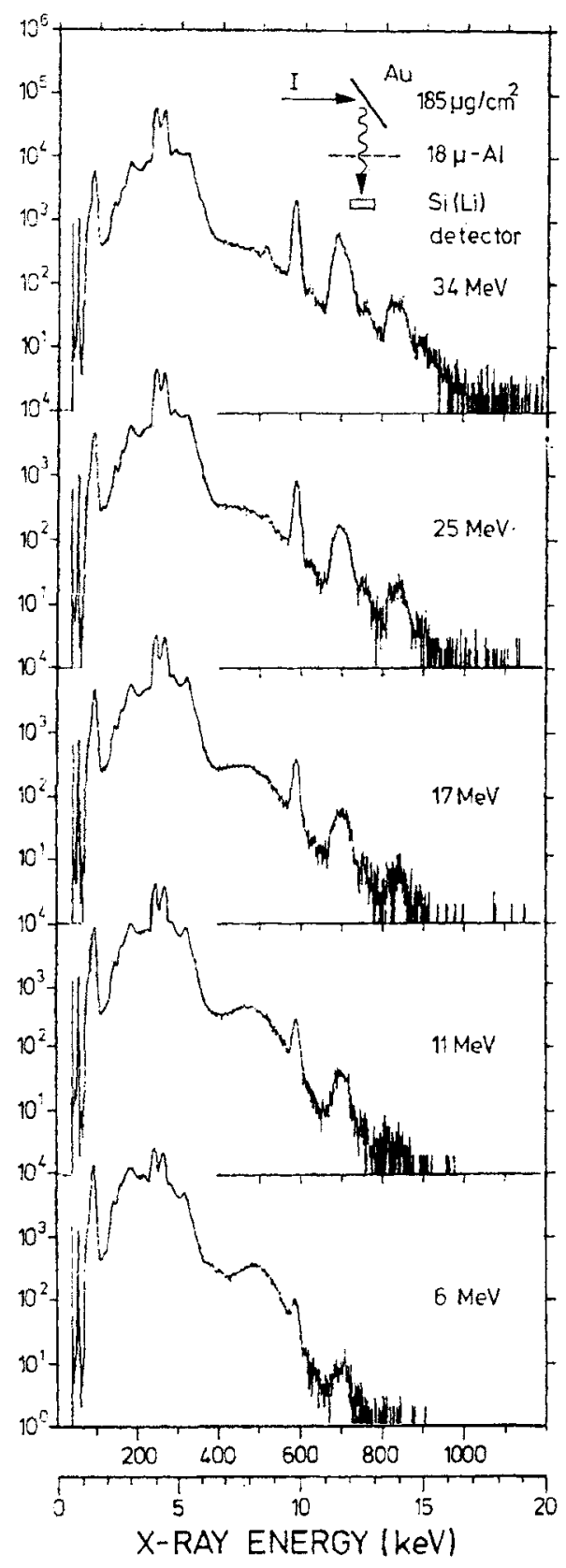

Fig. 6. X-ray spectra from $\mathrm{I} \rightarrow$ Au collisions at different bombarding energies. 


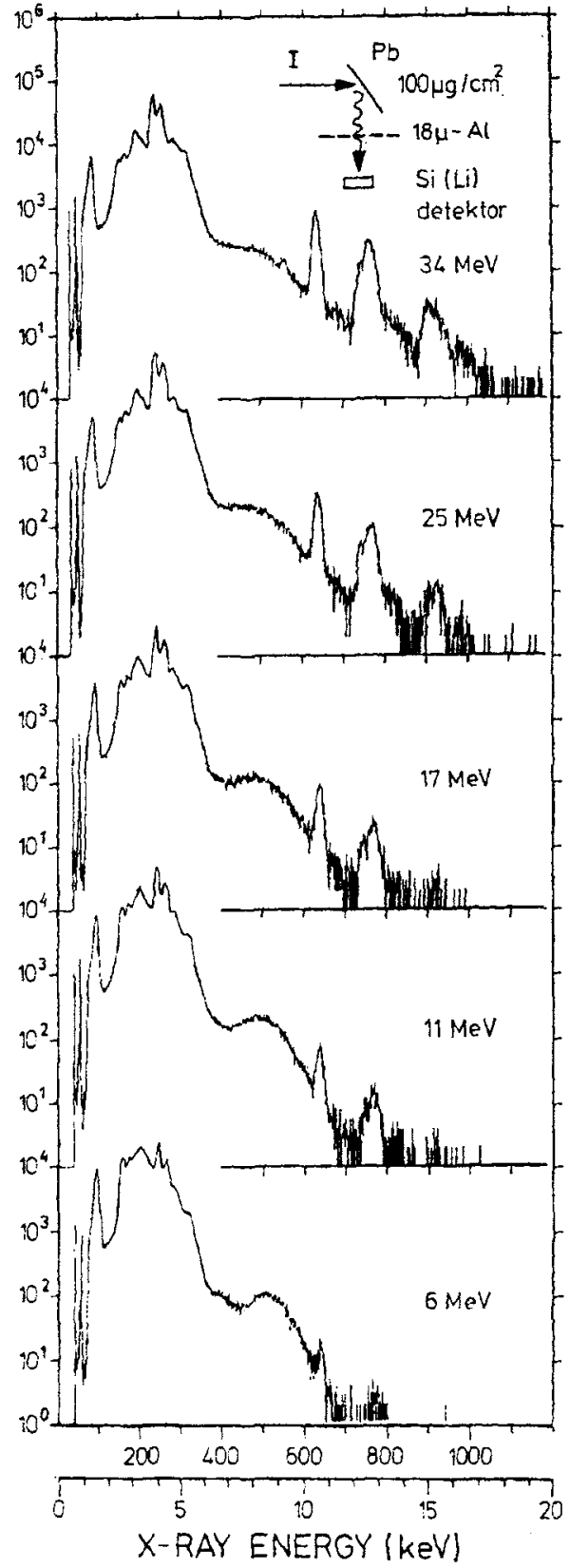

Fig. 7. X-ray spectra from $\mathrm{I} \rightarrow \mathrm{Pb}$ collisions at different bombarding energies.

calculated peak positions is within the accuracy of the measurements. It is the most striking experimental result.

The width of the quasiatomic structure depends linearly on the bombarding velocity and the extrapolation to $v \rightarrow 0$ gives a width smaller than $0.3 \mathrm{keV}$. Such a dependence points to a $(1 / v)$-transition time through the quasiatom. From $\Delta E=h\left(v / 2 R_{\mathrm{min}}\right)$, a lower limit of the size of the system can be estimated. The value obtained $0.9 \times 10^{-10} \mathrm{~cm}$ is factor of 4 smaller than the radius of the $M$ shell. This distance is reached in head-on collisions but at energies larger than $12 \mathrm{MeV}$, whereas the peak structure already appears at $6 \mathrm{MeV}$.

The ratio of cross sections $\sigma_{z} / \sigma_{1}$ only weakly depends on the velocity $\left(\sim v^{0.7}\right)$. This weak dependence is to be expected according to eq. (11a), if a two-collision mechanism is assumed, and if predominantly spontaneous radiation is emitted.

The absolute cross sections (Fig. 10) give a value $\sigma_{z} / \sigma_{1}$ of about $10^{-2}$ instead of $1.6 \times 10^{-4}$ according to eq. (11 a). Either the time between successive collisions $\tau_{12}$ or the radiative life-time of the

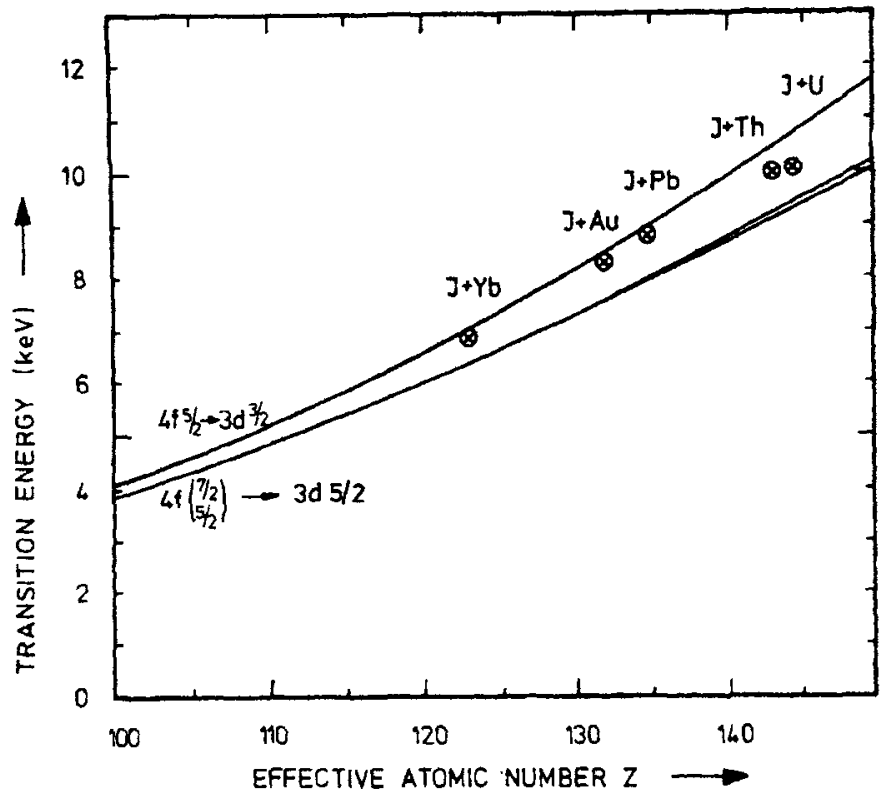

Fig. 8. Energy of peak position of the quasiatomic structure depending on the combined atomic number. For comparison, the calculated electronic $4 f-3 d$-transitions are given (ref. [12]).

vacancy $\tau_{m n}$ in the combined system must be reduced by a factor of about 50 compared with the estimates of equations $(8 \mathrm{a})$ and (11 a). The ratio $\sigma_{2} / \sigma_{1}=\left(\tau_{m n 1}^{\mathrm{rad}} / \tau_{18}\right) p$, with $p$ the crossing probability of the (3dit $\left.-3 p \frac{1}{2} \frac{1}{2}\right)$-level crossing has been measured as well. At $11 \mathrm{MeV}$ we obtain a value $\sigma_{2} / \sigma_{1}=4.6 \times 10^{-4}$. With $\tau_{m n} / \tau_{12}=2 \times 10^{-3}$ a value of the crossing probability $p=0.22$ is calculated. The high value of $\sigma_{Z} / \sigma_{1}$ cannot be explained by smaller values of $\tau_{12}$ otherwise $p$ had to have values larger than 1 . Only if the radiative lifetime in the quasiatom is reduced by a factor of 50 compared with the theoretically estimated values, are the measurements in agreement with the two-collision mechanism.

$\sigma_{2} / \sigma_{2}$ has a velocity dependence proportional to $v^{2}$ (Fig. 10). A dependence stronger than $v$ is to be expected, if $p$ increases with velocity. A Landau-Zener crossing shows an increasing crossing probability with increasing velocity. The experiments thus support

IMPACT ENERGY (MEV)

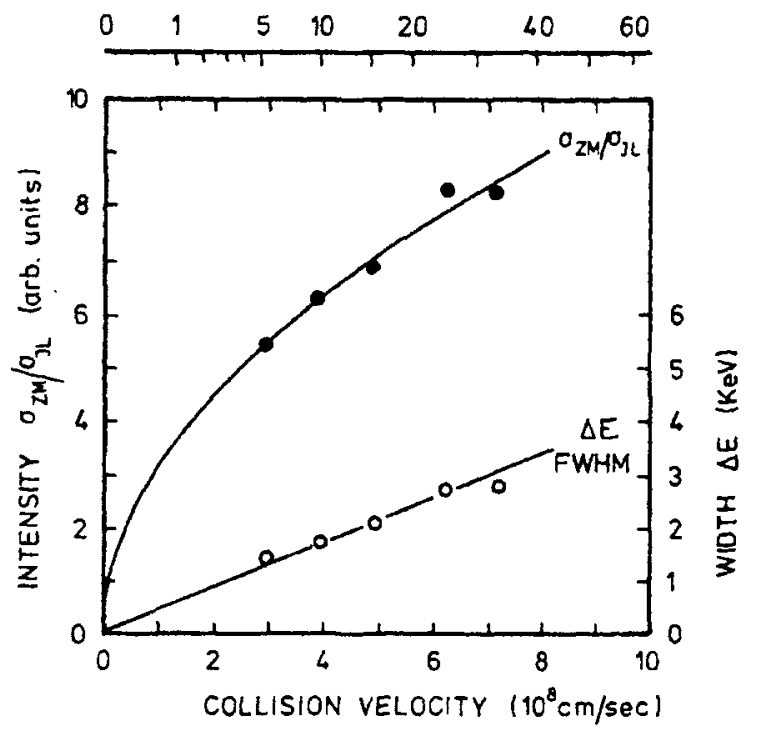

Fig. 9. Width and relative intensity of quasiatomic structure in $\mathrm{I} \rightarrow \mathrm{Au}$ collisions as a function of bombarding energy. 


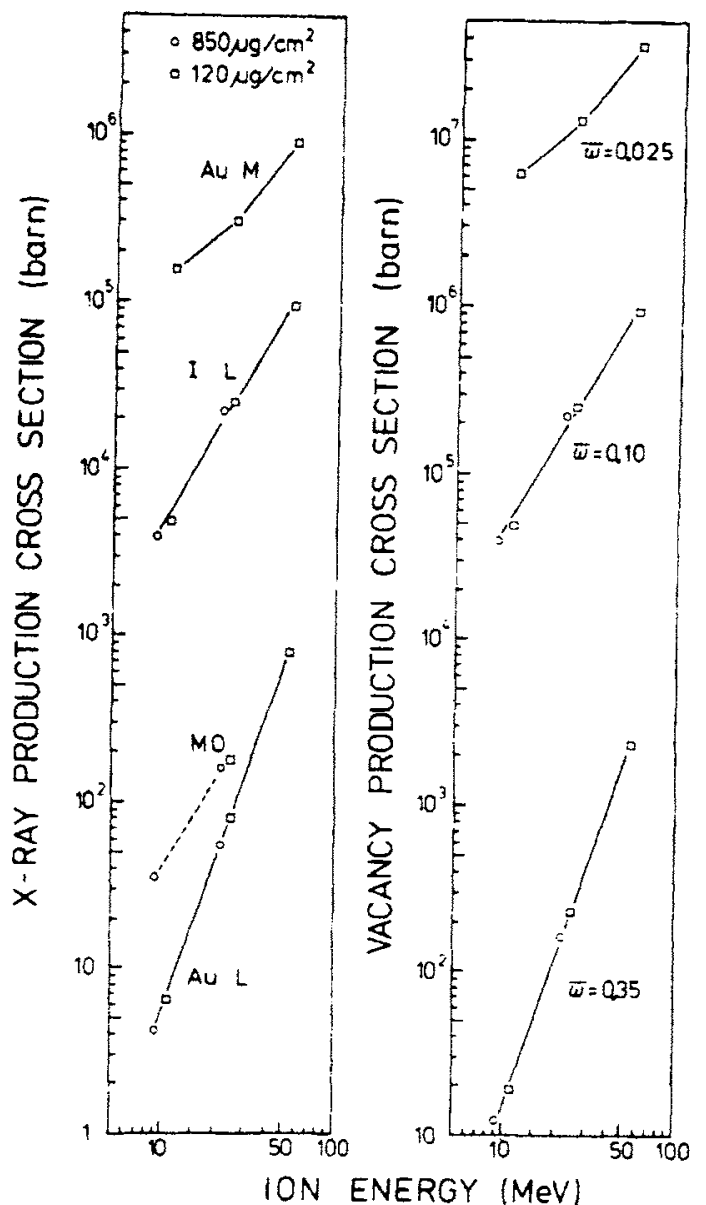

Fig. 10. Absolute cross sections for $\mathrm{I} \rightarrow \mathrm{Au}$ collisions as a function of bombarding energy (ref. [3]).

the existence and nature of the crossing in Fig. 4, which gradually is overcome at higher velocities. At low energies the crossing leading to higher $\mathrm{X}$-ray energies and finally to $L \mathrm{X}$-rays from the target is closed. The spectrum is cut off at energies higher than the $M$-energy of the combined system.

The very existence of this crossing may cause the break-off of the spectrum at energies larger than the quasiatomic $M \mathrm{X}$-rays. A comparable crossing does not exist for the $K$ - and $L$-shell of the quasiatom.

Assuming that all anisotropy stems from induced transitions, the strong anisotropy found seems to prove the existence of induced transitions. From the measured anisotropy value we obtain at $11 \mathrm{MeV}$ with eq. (14) a value $\sigma_{\mathrm{ind}} / \sigma_{\text {spon }}=0.4$, whereas according to eq. (12) a much smaller value of $5 \times 10^{-3}$ should be expected. The cross section for induced radiation seems to be increased by the very large factor of 4000 , whereas the spontaneous radiation seems to be increased by a factor of 30 . The increase of the spontaneous radiation may be partly due to a larger effective size of the quasiatom, which is indicated by Fig. 4. A value $8 \times 10^{-10} \mathrm{~cm}$ is still in agreement with the correlation diagram thus giving already a factor of increase of 11 .

The large factor of increase of the induced transitions cannot be understood within the model. The spontaneous transitions in the quasiatoms may be anisotropic as well, if the $m$-substates of the $4 f$-shell in the quasimolecule are not fully occupied. In that case the existence of induced transitions cannot be verified experimentally by an anisotropy measurement.

The appearance of a line structure at low energies, its width, its anisotropy, and its large cross section, cannot be understood within the model discussed. The measurements call for a mech-
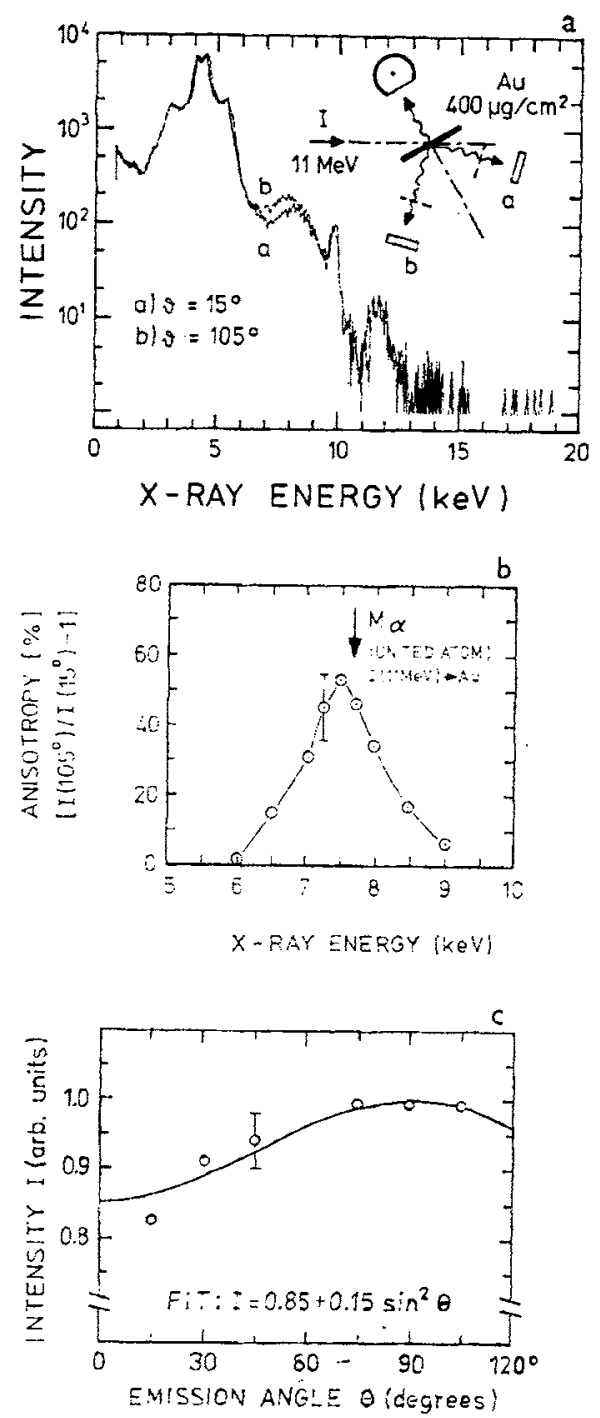

Fig. 11. Anisotropy of the quasiatomic structure $\left(7.1 \mathrm{keV}<E_{x}<9.5 \mathrm{keV}\right)$ at i1 $\mathrm{MeV}$ bombarding energy in $\mathrm{I} \rightarrow$ Au collisions.

anism, which produces even at low energies and small distances of approach high transition rates in the quasiatom. The open questions experimentally still to be investigated are:

(1) Is the two-collision mechanism really the only one, or may quasiatomic X-rays be produced also in single collisions?

(2) Can the assumption that the spontaneous radiation emitted from the quasiatom is isotropic be maintained?

(3) Which excitation mechanism leads to the predominant emission of quasiatomic $\mathrm{X}$-rays at small bombarding energies and distances of approach?

Differential measurements and further measurements of the anisotropy for solid and gas targets will give the information needed to further clarify the excitation mechanism.

The X-rays from quasiatomic systems allow, as the measurements presented show, the determination of inner shell energies of superheavy elements with an accuracy of about $2 \%$. Hope still exists of clarifying specific questions of the atomic structure of superheavy elements in heavy ion collision experiments without actually making the nuclei. The experiments are like exploratory missions to SHE-land without actually landing there. Should we land there, they will be forgotten. However, they may be our only glimpse of the new Atlantis, on whose the existence our theoreticians so convincingly insist. 


\section{References}

1. Mokler, P. H., Stein, H. J. and Armbruster, P., Phys. Rev. Lett. 29, 827 (1972).

2. Mokler, P. H., Stein, H. J. and Armbruster, P., in Proceedings of the International Conference on Inner-Shell Ionization Phenomena, Atlanta, Georgia, 1972, CONF-72040, Vol. 2, 1283, 1973.

3. Stein, H. J., in GSI 73-11, 106 (1973)

4. Gove, H. E., Jundt, F. C. and Kubo, H., Bull. Am. Phys. Soc, 18, No. 4, 559 (1973); Jundt, F. C. and Gove, H. E., Proc. Int. Conf. on Nuclea! Physics, Munic, 1973, Vol. 2, p. 138.

5. Maller, B., Smith, K. and Greiner, W., Phys. Lett, 49B, 219 (1974).

6. Saris, F. W., von der Weg, W. F., Tawara, H. and Laubert, R., Phys. Rev. Lett. 28, 717 (1972).

7. Lichten, W., Phys. Rev. 164, 131 (1967).

8. Barat, M. and Lichten, W., Phys. Rev. A 6, 211 (1972).

9. Armbruster, P., Z. Phys. 166, 341 (1962).

10. Specht, H. J., Z. Phys. 185, 301 (1965).

11. Stein, H. J., Lutz, H. O., Mokler, P. H. and Armbruster, P., Phys. Rev. A 5, 2126 (1972).

12. Fricke, B. and Soff, G., in GSI-T1-74 (1974).

13. Meyerhof, W. E., Saylor, T. K., Lazarus, S. M., Little, W. A., Triplett, B. B. and Chase, Jr, L. F., Rev, Lett. 30, 1279 (1973).

14. Greenberg, J. S., Proc. Int. Conf. on Nuclear Physics, Munic, 1973, Vol. 2, p. 82.

15. Gippner, P., Kaun, K.-H., Stary, F., Schulze, W. and Tretyakov, Yu. P., JINR, E7-7536. Dubna, 1973.

Gesellschaft für Schwerionenforschung $M B H$

Postfach 541

6100 Darmstadt 1, BRD 